\title{
$\mathrm{dR}_{\text {Revisand a Filiosofia }}^{\text {esonanias }}$
}

Número 3 - Junio/Año 2017, pp.9-25

\section{Ensayo Introducctorio a los Pluralismos Epistemológicos en Filosofía de la Ciencia}

\author{
INTRODUCTORY ESSAY ON THE EPISTEMOLOGICAL \\ PLURALISMS IN PHILOSOPHY OF SCIENCE
}

FECHA DE RECEPCIÓN: 30/08/2016 - FECHA DE ACEPTACIÓN: 23/12/2016

\author{
Gonzalo Aguilera Retamal ${ }^{1}$ \\ gonzlar@gmail.com \\ Universidad de Chile
}

\begin{abstract}
Resumen
En torno al tópico de las relaciones interteóricas existen dos posturas: por un lado, está el reduccionismo, el cual supone que dentro de una disciplina científica siempre existirá reducción de la teoría más débil explicativamente a la teoría más fuerte y, por el otro, está el pluralismo que niega la tesis reduccionista. El presente artículo tiene como objetivo general profundizar en la estructura conceptual de la postura pluralista y se divide en tres partes cuyas metas son: primero, presentar antecedentes intelectuales poco conocidos de la postura epistemológica pluralista, así como también algunos de sus argumentos para su defensa. Segundo, desarrollar los dos contextos en filosofía de las ciencias en los cuales el pluralismo aparece como una solución posible en contraste con el monismo y el reduccionismo. Tercero, la posibilidad de establecer la necesidad de la relación entre inconmensurabilidad y pluralismo para definirlo como una postura que se opone al reduccionismo como un hecho necesario del desarrollo de las ciencias.
\end{abstract}

Palabras clave: pluralismo, reduccionismo, monismo, relaciones interteóricas, inconmensurabilidad.

\begin{abstract}
There are two positions regarding the topic of inter-theoretical relations: on the one hand, reductionism, that supposes that within a scientific discipline there will always be a reduction from the weakest explanatory theory to the strongest one, and, on the other hand, pluralism, that denies the reductionist account. This article has as general objective "to deepen into the conceptual structure of the pluralist position» and is divided in three parts. The first part intends to present a couple of little-known intellectual background of the pluralist epistemological stance, as well as some arguments for its defense. The second part will develop both contexts in philosophy of science in which pluralism as philosophical stance appears as a possible solution in contrast to monism and reductionism. The third part will deal with the possibility of establishing the necessity of the relationship between incommensurability and pluralism, to define it as a position opposed to reductionism as a necessary fact for the development of science.
\end{abstract}

Key words: pluralism, reductionism, monism, intertheoretic relationships, incommensurability.

\footnotetext{
${ }^{1}$ Licenciado en Filosofía, Universidad de Chile. Magíster en Estudios Cognitivos, Universidad de Chile.
} 


\section{Introducción.}

En filosofía de las ciencias, la cuestión de cómo se relacionan entre sí dos teorías en competencia dentro de una disciplina científica cualquiera o -lo que es análogo, el tema de las relaciones interteóricas- lejos de ser un tópico resuelto en todas sus dimensiones no tiene una respuesta única, sino más bien, se pueden distinguir dos respuestas bien diferenciadas: por un lado, aquella que supone que toda relación interteórica a la larga lleva a la subsunción de las teorías explicativamente más débiles en el corpus teórico de las más poderosas, respuesta que puede denominarse reduccionista, y por otro, aquella que niega esta primera respuesta, la que puede denominarse como pluralista, pero cuyo contenido conceptual tiene diversos matices que serán explorados en el presente ensayo a la luz de otros conceptos filosóficos de la ciencia que servirían de apoyo.

Sobre esto último en particular, considero que la noción de inconmensurabilidad, en conjunción con las de holismo y subdeterminación, son las responsables de proveer el contenido conceptual esencial que caracterizaría las diferentes posturas epistemológicas pluralistas posibles. Por mor de no extenderme en este punto, la relación será simplificada del siguiente modo: si en cierto momento dado, sobre un mismo fenómeno $\mathrm{F}$ cualquiera tenemos dos teorías T1 y T2 en competencia que comparten similar poder explicativo y predictivo, pero carecemos de un modo de conmensurar ${ }^{2}$ entre ellas, entonces tanto T1 como T2 deben ser conservadas como válidas para explicar F. Esta idea será desarrollada específicamente en la tercera parte del artículo, el cual en su conjunto no argumenta en función de convencer a aquellos filósofos de la ciencia que se consideran reduccionistas, sino que, más bien tiene como objetivo desarrollar la profundidad de la postura pluralista, la cual es, desgraciadamente, a veces asociada con laxitud conceptual.

La estructura del artículo tiene tres partes, cuyos objetivos específicos son: Primero, exponer brevísimamente algunos de los antecedentes intelectuales del pluralismo anteriores a las ideas de Kuhn y Feyerabend, así como algunos de los argumentos generales que sostendrían al pluralismo en filosofía de las ciencias. Como se expone en el párrafo anterior estos argumentos generales servirán más como una síntesis de aquella argumentación que sostiene el filósofo pluralista, que como una apelación vehemente al abandono del reduccionismo. Segundo, apoyándose en un desarrollo relativamente contemporáneo que "estandariza" la definición de pluralismo, se utilizará una versión

\footnotetext{
${ }^{2}$ En el presente artículo se utilizará una de las nociones de Kuhn de conmensurabilidad, la cual será explicitada en la sección correspondiente. Empero, nótese que se trabajará de un modo que difiere del original, ya que se usará para hablar de teorías y no necesariamente de paradigmas.
} 
simplificada que ponga en contexto cómo éste, es la postura antagonista al monismo respecto a ciertos problemas clásicos de la filosofía de la ciencia. Tercero, aprovechando lo desarrollado en la sección anterior, se ensayará respecto a la posibilidad de hablar de diferentes clases de pluralismos, cuestión que permitirá avanzar en la precisión de la postura pluralista.

\section{I.- Antecedentes intelectuales del pluralismo y algunos argumentos para su de- fensa.}

Es común que la noción de pluralismo en filosofía de las ciencias sea considerada una consecuencia del historicismo kuhniano (Thomas Kuhn, 1970) y sea equiparada, conceptualmente, a la postura del "anarquismo epistemológico" desarrollada por Feyerabend (1975), no siendo difícil encontrar trabajos contemporáneos locales que traten tales relaciones de equivalencia en contenido filosófico (Marisol Facuse, 2003; Andrés Osorio, 2011).

No obstante, es posible encontrar antecedentes intelectuales al pluralismo antes de la segunda mitad del siglo XX, periodos en que se dan, tanto la acometida historicista de Thomas Kuhn, como la anarquista epistemológica de Paul Feyerabend. El "pluralismo", entendido como actitud filosófica puede ser documentado como presente en el pensamiento de la física de la primera mitad del siglo XX, mientras que el holismo de Duhem (1906) es un antecedente bastante claro de cómo Kuhn considera que se relacionan las evidencias empíricas y los paradigmas, condición sine qua non para desechar la postura que supone un marco objetivo neutral para dirimir entre dos paradigmas. Esto conduce a establecer la existencia de períodos donde, precisamente, existiría una convivencia entre diferentes paradigmas sin hegemonía de ninguno de ellos.

Alisa Bokulich en su relativamente reciente obra, Reexamining the Quantum-Classical Relation: Beyond Reductionism and Pluralism (2008), presenta al pluralismo teórico en el contexto del problema de las relaciones interteóricas entre mecánica clásica y cuántica, donde tales teorías pueden ser tomadas como relacionándose reductivamente, es decir, una teoría como derivada de la otra, o pluralistamente, donde cada teoría tiene su dominio distintivo de leyes, entidades y conceptos, sin posibilidad de reducción ${ }^{3}$.

Como se planteó antes, la tesis de la inconmensurabilidad de Kuhn y Feyerabend

\footnotetext{
${ }^{3}$ Quizás, vale la pena apuntar que el pluralismo también habría sido defendido en el contexto de la psicología. Bokulich nombra a Fodor (1974), quien argumenta que la psicología no puede ser reducida a las neurociencias. Luego, según Fodor, la psicología es y siempre será una ciencia autónoma sin relación a las neurociencias. Ahora bien, para no abundar en el contenido y las muchas ramificaciones que se pueden extraer del pluralismo aplicado al estudio del fenómeno mental y cognitivo, dejo en esta nota constancia que el pluralismo puede considerarse como postura filosófica anterior y más general, al autonomismo que postula la existencia de "niveles descriptivos" o "de análisis" para cualquier fenómeno.
} 
-aunque cada uno con diferentes sentidos- habría jugado históricamente un rol central en el movimiento desde el reduccionismo al pluralismo teórico. Según Bokulich, autores posteriores como John Dupré derivarían de esta postura pluralista, estrictamente epistemológica, lo que se denominará "realismo promiscuo". Sintéticamente apuntado, esta clase de realismo sería aquel que sostiene que el problema no es que las taxonomías científicas fallen en capturar las clases naturales reales, sino que todas ellas lo hacen correctamente, multiplicándose así tales clases naturales.

Más allá de la cuestión de la metafísica de las ciencias, es decir, de si el pluralismo necesariamente implica un realismo promiscuo, lo interesante es que la filósofa e historiadora de las ciencias expone cómo, en el progreso de su física, el afamado Werner Heisenberg habría mantenido una actitud filosófica pluralista frente a la relación entre mecánica newtoniana y mecánica cuántica. El argumento de Bokulich se basa en una revisión de la propia filosofía de las ciencias de Heisenberg, la cual estaría disgregada en diversas obras del científico.

Muy resumidamente, tal actitud pluralista existiría en la, fuertemente original, visión de filosofía de las ciencias del físico alemán, basada en la noción de teoría cerrada, expuesta ya en 1927, y que la definiría como aquel sistema tejido apretadamente de axiomas, definiciones y leyes que proveen una descripción perfectamente acertada y definitiva de un dominio acotado de un fenómeno. Una "teoría cerrada" se distinguiría de una "teoría fenomenológica" dentro de la filosofía de Heisenberg en que la primera sería una descripción verdadera de lo que el mundo es, mientras que la segunda no.

Luego, Bokulich apunta que Heisenberg no considera ni la mecánica cuántica ni la mecánica newtoniana como teorías fenomenológicas, adoptando así una interpretación realista de ambas. El sistema de definiciones y axiomas de la mecánica newtoniana describiría una estructura eterna de la naturaleza. Por consiguiente, la única manera de interpretar este realismo de Heisenberg sobre ambas mecánicas sería una salida pluralista, la cual sería apoyada por su idea de diferentes regiones de la realidad adecuadas a cada una de sus "teorías cerradas". Más exactamente, la postura del físico podría considerarse como un "pluralismo metafísico nomológico", existiendo así no reducción, sino que conservación de ambas teorías.

Las teorías cerradas de Heisenberg exhibirían una clase de comportamiento holístico, dado que al ser altamente interdependientes entre sí los elementos que las componen, sería entonces, imposible la modificación de un solo elemento sin cambiar el todo de las teorías cerradas. Según Bokulich: "El compromiso de Heisenberg con el holismo lo lleva a adoptar un modelo del cambio de las teorías revolucionaria y antigradualista 
-en muchos aspectos semejante al de Kuhn" ${ }^{4}$ (Alisa Bokulich, 2008: 35).

Pues bien, en esta línea es importante notar que existe una dependencia directa entre la tesis del holismo y la del pluralismo, ya que el pluralismo aparece ligado siempre a visiones donde las teorías son consideradas de manera contraria a un sistema axiomático de proposiciones; el pluralismo aparece ligado a la visión de las teorías como conjuntos de proposiciones interdependientes, que se comportan como un todo al contrastarse con el mundo, es decir, confirmándose de modo holístico.

Empero, aunque es sabido que tanto Kuhn como Feyerabend sostenían -quizás no tan explícitamente como sería lo deseable- tal holismo de la confirmación, considero que la relación entre holismo y pluralismo ha sido subestimada. Por lo tanto, además de la necesidad de reivindicar a Heisenberg como antecedente intelectual del pluralismo, la presencia de Pierre Duhem me parece que debe ser agregada a la figura del físico alemán, en tanto que es él, quien desarrolla la primera versión del holismo delimitada a la física y que, posteriormente, Quine (1951) extendería para toda la ciencia. Centrándome en Duhem, él dirá ya en 1906:

En resumen, el físico nunca puede someter a una hipótesis aislada a prueba experimental, sino que únicamente al todo de un grupo de hipótesis; cuando el experimento está en discrepancia con sus predicciones, lo que él aprende es que al menos una de las hipótesis constitutivas del grupo es inaceptable y debiese ser modificada; pero el experimento no determina cuál debiese ser cambiada (...) La física no es una máquina que se deja desmontar; no podemos tratar cada pieza aisladamente y, de modo de ajustarla, esperar hasta que su solidez haya sido cuidadosamente verificada. La ciencia física es un sistema que debe ser tomado como un todo; es un organismo en el cual una parte no puede ponerse a funcionar, excepto cuando las partes que están más lejos de ésta son llamadas a actuar, algunas más que otras, pero todas en algún grado (Pierre Duhem, 1906: 8).

Por lo tanto, es la conjunción de las ideas de que: primero, las teorías se confirman como un todo y segundo, que dos teorías mutuamente incompatibles pueden explicar los mismos acontecimientos observables (i.e. subdeterminación de la teoría científica), la base que permitirá a Kuhn a hablar de inconmensurabilidad ${ }^{5}$.

\footnotetext{
${ }^{4}$ Todas las citas del presente artículo tienen fuentes en inglés, y todas han sido traducidas por su autor. En caso de que se quiera leer textualmente alguna de ellas, entonces se debe acudir a la obra y página indicada al final de cada cita.

${ }^{5}$ Nótese que esta continuidad que estoy estableciendo desde el holismo moderado de Duhem, pasando por la tesis de DuhemQuine, la subdeterminación de las teorías científicas, la inconmensurabilidad y el pluralismo se circunscribe sólo al ámbito de lo que puede establecerse por el puro contenido conceptual de las ideas apuntadas. La cuestión histórica de cómo cronológicamente de hecho se dieron tales relaciones entre filósofos es bastante más compleja e involucra un trabajo que aquí solamente señala algunas pistas para aquel que quiera, exhaustivamente, adentrarse en ello.
} 
Ahora bien, dejando de lado detenernos en las ideas de pluralismo y argumentos a favor de éste, que pueden extraerse de autores como Kuhn y Feyerabend, -quienes son más clásicamente ligados a tal postura epistemológica- como he mencionado en la introducción, considero conveniente apuntar a los argumentos más sintética y contundentemente postulados a favor del pluralismo epistemológico. Esta síntesis argumentativa se da en el discurso presidencial de Patrick Suppes de 1978 (tres años después de la publicación de Against Method (1975) de Feyerabend) en la Philosophy of Science Association, donde reivindicó que el tiempo de defender a la ciencia contra la metafísica ya habría pasado -siendo esta defensa la causa original por el movimiento que buscaba la unidad de la ciencia. El ataque de Suppes contra la empresa de unificación está constituido por un puñado de argumentos bien definidos:

- Primero, no hay posibilidad de encontrar un lenguaje común que englobe todas las ciencias. Esto se puede constatar en el hecho de la especialización y el crecimiento de los términos técnicos de las disciplinas, las cuales tienden a la divergencia más que a la convergencia. En efecto, las ciencias nunca han atravesado un periodo de simplificación del lenguaje, como puede verse en la más antigua de las ciencias cuantitativas, la astronomía.

- Segundo, no tiene sentido una unificación apelando a cierto "substrato común" al cual todo puede reducirse. Suppes apela a una analogía: así como sucede que dos generaciones alejadas de computadoras pueden llevar a cabo las mismas tareas, pero bajo diferentes implementaciones materiales $-\mathrm{v}$. gr. válvulas termoiónicas versus semiconductores-, puede suceder lo mismo con las ciencias, es decir, que más allá de la igualdad en la superficie conceptual, tal reducción sea empíricamente vacía. Yendo a un caso concreto, se aduce que es universalmente aceptada la imposibilidad de tratar de resolver los problemas de la química cuántica apelando solo a leyes fundamentales de la mecánica cuántica.

- Tercero, no existe ninguna unidad en los métodos de las ciencias, más allá de lo trivialmente obvio, tal como el uso de la matemática elemental, siendo "elemental" desde la perspectiva de las mismas matemáticas, o ciertos ideales metodológicos que nunca son perfecta y uniformemente logrados, como la falsabilidad y reproducibilidad. La falta de unidad de métodos es fácil de constatar interdisciplinariamente, pero incluso intradisciplinariamente, para los expertos en una disciplina el detalle de los métodos experimentales de una subdisciplina puede llegar a ser ininteligible. Por ejemplo, físicos que trabajan en física de estado 
sólido, pueden no comprender el trabajo de aquellos que trabajan en teoría de cuerdas. Incluso, lo mismo tiende a ocurrir en disciplinas menos desarrolladas como la psicología.

- Cuarto, no hay medida física posible de ser tomada con certidumbre cabal. El desarrollo de la mecánica cuántica en el siglo XX llevó renuentemente a los físicos, pero de modo conclusivo, a reconocer que no tiene sentido afirmar que cualquier cantidad física puede ser medida con un grado arbitrario de precisión en conjunción con la medición simultánea de otra variable física. El reconocimiento de tal inhabilidad para efectuar mediciones exactas no se debió a inadecuaciones técnicas, sino que está ínsita en la misma teoría física.

- Quinto, y análogamente a la conclusión anterior, puede verse el fracaso en los intentos de buscar completitud en aritmética con el trabajo de Gödel o de la incompletitud de la teoría de conjuntos, la cual puede ser construida utilizando diversos axiomas independientemente; $-\mathrm{v}$. gr. la hipótesis del continuo es independiente al axioma de elección.

La conclusión a que llega Suppes (1978) la expone mediante una imagen; la ciencia no es una serie convergente, cuyo límite tiende a una teoría final, sino que es divergente. Es una empresa de perpetua solución de problemas, donde no existe área de la experiencia que esté totalmente asentada en un conjunto de verdades básicas. Estaríamos más bien, continuamente confrontados a nuevos problemas y situaciones, los cuales trataríamos con un popurrí de métodos científicos, técnicas y conceptos ad hoc. En esta visión de la ciencia la verdad objetiva no habría desaparecido directamente, pero lo que nosotros llamaríamos una "visión global o cosmológica de la verdad" sería mirada con escepticismo para dar paso a la visión pluralista de la ciencia.

\section{II.- Acercándose a una definición de pluralismo epistemológico.}

A partir de lo expuesto anteriormente se devela en efecto, en filosofía de las ciencias, la existencia de dos grandes problemáticas separables, aunque muy estrechamente conectadas,

(A) Sobre la unidad de las ciencias;

(B) Sobre las relaciones interteóricas o cómo, usualmente, dentro de un mismo dominio 
de fenómenos compiten entre sí teorías que tienen el mismo -o muy similar- poder explicativo y/o predictivo.

Así pues, se definen, preliminarmente, monismo y pluralismo como las respuestas antagónicas o los extremos sobre estas dos cuestiones. El monismo es la postura que responde afirmativamente a la cuestión (A), la existencia de una unidad en las ciencias, y el (reduccionismo) es el tipo de monismo que respondería afirmativamente a (B), es decir, a la existencia necesaria de reducciones interteóricas. El pluralismo sería la respuesta negativa a (A) y (B). Sin embargo, es menester apuntar que, aun cuando lo recién dicho sobre el monismo parezca en su contenido intuitivamente unívoco, no existe unidad conceptual común a todos los monistas epistemológicos.

Por ejemplo, la cuestión (A) es bien conocida por haber sido perseguida por los positivistas lógicos a principio del siglo XX. El Círculo de Viena, el grupo más prominente de positivistas lógicos, tuvo su periodo de auge entre 1920 y 1930 y, contrariamente a pensadores anteriores, no eran exclusivamente filósofos abocados al pensar sobre la ciencia, sino que muchos de sus representantes eran científicos, como, por ejemplo, Moritz Schlick quien era físico o Hans Hahn que era matemático. En un comienzo la constitución del Círculo parecía, más bien, un centro de reunión que un movimiento organizado, sin embargo, al advertir que compartían intereses afines respecto de cierto conjunto de problemas y una actitud común frente a éstos, los miembros del Círculo comenzaron a reunirse con asiduidad para discutirlos. Este proceso dio lugar en 1929 a la publicación de un manifiesto titulado Wissenschaftliche Weltauffassung, Der Wiener Kreis -"Concepción científica del mundo, Del Círculo de Viena"- que hacía exposición breve de la postura filosófica del grupo.

Pues bien, dentro del mencionado manifiesto sobre los objetivos del Círculo de Viena resulta atingente registrar que lo que se entendía como la investigación de una "concepción científica del mundo" era la búsqueda del logro de una ciencia unificada, o sea, conciliar los resultados de los investigadores individuales con los demás campos de la ciencia. A esto siguió la necesidad de la construcción de un sistema formal neutral, un simbolismo libre del lenguaje histórico y un sistema total de conceptos. Con esta herramienta la concepción científica del mundo no conocería enigmas sin resolver, ya que sería la precisa para demarcar entre aquello que es posible de descubrir empíricamente, de lo que es un sinsentido metafísico.

Esta empresa de unificación tendría su representación más canónica en los primeros desarrollos filosóficos de Rudolf Carnap, quien en su artículo Überwindung der Metaphysik durch logische Analyse der Sprache (1931) -"La superación de la metafísica me- 
diante el análisis lógico del lenguaje"- explicita cómo el principio de verificabilidad ${ }^{6}$, permitiría liberar a las proposiciones del lenguaje científico de la metafísica, dando lugar así a una primera fase de construcción de un lenguaje con categorías sintácticas capaces de no originar errores como secuencias verbales sin sentido, las cuales pueden generarse en el lenguaje natural sin violar regla sintáctica alguna.

De lo último apuntado basta un ejemplo; considérese la oración "César es un número primo". Tal oración no viola ninguna regla de sintaxis del lenguaje natural, ni tampoco está compuesta de algún pseudoconcepto (un elemento que no puede ser verificado por sí solo), pero sin embargo es claro que es inverificable. Luego, se hace imperiosa la construcción de un lenguaje para la ciencia que impida esta clase de pseudoproposiciones. Ergo, muy sintéticamente dicho, la empresa de unificación carnapiana estaría guiada por un principio que trascendería las disciplinas, y que tendría como resultado un sistema que puede ser descrito a priori en sus componentes formales.

Al contrario de Carnap, el filósofo Otto Neurath, también participante del Círculo de Viena, tenía una teoría de la unificación de la ciencia circunscrita a una acción más práctica, a saber, el reunir a los científicos de distintos campos de la ciencia de modo de facilitar la comunicación e interconexión. Neurath pensaba que una determinación $a$ priori de las formas de interconexión sería imposible, luego, en vez de buscar una síntesis de las diferentes ciencias sobre la base de una filosofía independiente y apriorística, las ciencias debiesen ir con el tiempo desarrollando sus propias interconexiones, una sistematización sin ningún resultado específico preconcebido (Potochnik, 2011). Por ende, el monismo respecto a la cuestión (A), ha tomado diversas formas con el tiempo, de las que solamente he explicitado dos de sus más representativas a principios del siglo XX.

Respecto a la cuestión (B) acaece la misma situación que se ha delineado en torno al problema de la unidad de las ciencias, es decir, que tampoco ha habido acuerdo sobre el modo en cómo se reduce una teoría a otra. Quien mejor lleva a cabo este contraste entre diferentes maneras de llevar a cabo reducciones es Nickles (1973), quien explora la existencia de dos conceptos de reducción que explicarían diferentes clases de relaciones interteóricas reductivas.

Nickles establece que, por una parte, existiría una "reducción ${ }_{1}$ ", la cual sería de un

${ }^{6}$ Los positivistas lógicos no acuden a ninguna entidad abstracta independiente de la experiencia para individualizar el componente significativo de un enunciado. Una proposición es verdadera mientras dé cuentas de un "estado de cosas", y falsa si no lo hace. Verificar consiste en acudir a este "estado de cosas" y evaluar si lo que está ahí es lo que enuncia la expresión. $\mathrm{Si}$ es el caso que el enunciado carece de valores veritativos por los cuales decidirse, entonces es asignificativo. Así pues, para los positivistas lógicos el conocimiento estaría fundamentado en enunciados elementales que conciernen a hechos simples, a saber, los "enunciados protocolares". Empero, esta descripción es más bien una "interpretación estándar" para entender fácilmente el principio de verificabilidad más que una exposición verídica de la opinión del Círculo, donde había fuertes desacuerdos en nociones básicas como "estado de cosas", "lo dado" o "enunciado protocolar". 
tipo "derivacional", donde T2 reduce1 a T1 sí y sólo si T2 explica T1, entendiéndose tal explicación como una derivación lógica mediante leyes puente. Esta noción de reducción sería original de Ernest Nagel -uno de los filósofos de la ciencia más famosos del siglo XX cercano al positivismo lógico-, para quien la reducción de este tipo conllevaría compatibilidad lógica entre T1 y T2, permitiendo una economía ontológica al combinarse los dominios de las teorías que entran en una relación de reducir una a la otra. En el artículo se menciona como la reducción de la teoría cinética a la mecánica estadística, o la de la óptica a la teoría del electromagnetismo, serían casos clásicos de reducción . $_{\text {. }}$

Por otra parte, habría una "reducción ${ }_{2}$ ", la cual no sería del tipo "derivacional" en el sentido lógico de Nagel -aunque sí lo sería en su sentido matemático más amplio- y donde $\mathrm{T} 2$ reduce $\mathrm{T} 1$ syss $\mathrm{T} 1$ pudiendo ser recuperado aproximadamente como caso especial o "límite" de T2. El acontecimiento que se considera paradigmático es la relación entre la teoría especial de la relatividad y la mecánica clásica, donde la primera podría ser "ajustada" de modo de subsumir a la segunda, por ejemplo, considerándose la mecánica clásica como la aplicación de la teoría especial de la relatividad en casos de velocidades muy menores respecto a la velocidad de la luz. Las desventajas que tendría la reducción ${ }_{2}$ respecto a la reducción de derivación lógica es que no se lograría economía ontológica, ni tampoco posibilitaría una comprensión del porqué T1 sería explicativa, aun cuando permite visualizar el proceso de reemplazo de T2 sobre T1.

Ahora bien, ya que el monismo es una postura sobre dos problemáticas diferentes y que, además, puede tomar distintas estrategias de salida, entonces son concebibles variadas clases de monismo. Simplificando, considero que respecto a (A) la postura de Neurath representa el monismo límite justo antes de pasarse a pluralismo, es decir, aquel monismo que se postula como cierta esperanza de unificación mediante la comunicación entre disciplinas, mientras que respecto a (B) la reducción ${ }_{2}$ representa también el monismo -que en este caso debe llamarse "reduccionismo"- que tendería a estar más cerca del pluralismo.

Tomando en cuenta que lo desarrollado anteriormente permite visualizar la existencia de dos tipos amplios de monismo pluralismo, así como también, cierta gradación entre los extremos, entonces, se vuelve imprescindible en este punto del artículo, avanzar desde las definiciones preliminares de "monismo" y "pluralismo" apuntadas al comienzo de esta sección, hacia la búsqueda de cierta definición que englobe, tanto lo esencial a todo pluralismo, como las diferentes versiones de pluralismo.

En torno al tópico del pluralismo epistemológico, hace una década que existe una 
definición tratando de capturar cierto consenso respecto a cómo definir el extremo del monismo, lo cual permite, a su vez, entender al pluralismo como el opuesto de tal definición basada en rasgos. Cito in extenso tal lista de rasgos del monismo expuesta por Kellert, Longino y Waters (2006):

1. El objetivo definitivo de la ciencia es establecer una visión única, completa y comprehensiva del mundo natural (o de la parte del mundo investigada por la ciencia) basada en un único conjunto de principios fundamentales

2. La naturaleza del mundo es tal que puede, al menos en principio, ser completamente descrita o explicada por tal visión.

3. Existe, al menos en principio, métodos de investigación que, si son correctamente seguidos, conducirían a tal visión.

4. Los métodos de investigación deben ser aceptados sobre la base de si ellos puedes conducir a tal visión;

5. Y, teorías y modelos individuales en ciencia deben ser evaluados en gran parte sobre la base de si ellos proveen (o están cerca de proveer) una visión comprehensiva y completa basada en principios fundamentales (Stephen Kellert et al., 2006).

Analizados detenidamente, considero que no todos estos rasgos son esenciales para la comprensión del monismo, al menos entendido como aquel posicionamiento que surge como circunscrito a las problemáticas (A) y (B), ya que, por ejemplo, la característica 2 tiene un innecesario compromiso metafísico sobre cómo es el mundo. Debido a que el énfasis del presente ensayo no va en línea con la cuestión del estatus ontológico de los objetos de las teorías, me parece innecesario asumir que tal rasgo es obligatorio en el monismo epistemológico. Me parece intuitivamente concebible que un filósofo se declare monista epistemológico respecto a la cuestión (A), sin necesidad de adscribir a cierta concepción del mundo. Expresando lo mismo, en otros términos, me parece compatible declarar que la ciencia tiende a la convergencia sin la necesidad de adscribir a tesis metafísicas. En este sentido la "convergencia" puede entenderse no necesariamente respecto a un mundo al que nos acercamos asintóticamente, sino que respecto a cómo convergerían entre sí las ciencias, o sea, más bien verse como una tendencia a la coherencia. Es obvio que parece más fácil ligar el monismo epistemológico a cierta preconcepción ontológica del mundo como una unidad estable, pero ello no parece que sea esencial.

Analizando los rasgos 3 y 4 puede decirse que es fácilmente rastreable en el monista con respecto a cierta teoría, es decir, en aquel que considera que sobre cierto fenómeno existe un método que está necesariamente ligado a su investigación, ergo, estos rasgos 
se corresponden mejor al monismo respecto problemática (B), aun cuando, dado que se conectan a los rasgos 1 y 2 en el contexto de Kellert et al. (2006) tratan sobre la problemática (A).

Efectivamente, si se retorna al rasgo 1, el cual me parece que es el único esencial para definir al filósofo monista que se sitúa afirmativamente frente a la existencia de alguna unidad de la ciencia, entonces pareciera ser que la lista de rasgos de Kellert et al. (2006) es útil para hablar e individualizar el pluralismo como posición respecto a la unidad de las ciencias -problemática (A)- pero, no arroja pertinentemente muchos detalles respecto a cómo entender una postura monista idéntica a alguna postura reduccionista -y, por extensión, comprender el pluralismo en la cuestión (B).

\section{Clases de pluralismos.}

Es necesario señalar que, en la sección anterior, aunque explicando que el primer rasgo de la lista de Kellert et al. (2006) sirve para definir el monismo respecto a (A), de lo que se sigue que es crucial para entender al pluralismo respecto a (A) como postura que niega tal primer rasgo, aún no se profundizó suficientemente respecto a la noción de pluralismo que aparece en torno a (B).

En la introducción y la primera parte del presente artículo quedó estipulado que hay de manera relativamente clara, en la noción de "inconmensurabilidad" conjuntamente con la del holismo y la subdeterminación de teorías científicas, base nocional suficiente para matizar la postura epistemológica pluralista. Consciente que el punto se abordó de manera general, en la presente sección se analizará con mayor particularidad, ya que resulta central para llevar a cabo la definición del pluralismo como contrario a reduccionismo.

La dependencia del pluralismo respecto al holismo se basa en lo siguiente: si no se sostiene el holismo, entonces ¿qué clase de visión respecto de la evolución de las teorías se tiene? Necesariamente, si no se apoya el holismo, entonces existirán experimentos cruciales -justamente aquellos que desestima la postura de Duhem (1906)- lo que permitiría ver la evolución teórica sobre cierto fenómeno, o dominio de fenómenos, como consistente en acumulación de conocimiento, y sucesivos reemplazos y subsunciones de las nuevas teorías respecto a las antiguas. Al contrario, dado que me parece verdadera la tesis del holismo, entonces la postura de Duhem permite sentar las bases de la versión más radical de Duhem-Quine, la cual está relacionada dentro de la filosofía de Quine con la subdeterminación de las teorías científicas (Gibson, 2004), lo que conlleva la po- 
sibilidad de que sobre un mismo fenómeno $\mathrm{F}$ cualquiera puedan existir dos (o más) teorías T1 y T2, en competencia, las cuales compartan similar poder explicativo y predictivo, aunque entre sí sean incompatibles. Esta incompatibilidad puede entenderse como cierta incomensurabilidad kuhniana en el sentido de la imposibilidad de encontrar un modo de traducir entre teorías que sea neutral a ambas. En términos de Kuhn:

Lo que está faltando no es la comparabilidad, sino que una unidad de medida en términos de la cual ambas [teorías] puedan ser medidas directa y exactamente. Al aplicar el término "inconmensurabilidad" a las teorías, únicamente he intentado insistir en que no hay un lenguaje común dentro del cual ambas podrían ser expresadas completamente y el cual, luego, podría usarse en una comparación punto por punto entre ellas (Thomas Kuhn, 1976: 191).

Pues bien, a partir del holismo, la subdeterminación y la inconmensurabilidad es posible sostener una visión no acumulativa del desenvolvimiento de las teorías científicas, visión que considera los casos de reducción como una cuestión contingente en la evolución de las teorías más que como un hecho necesario de las ciencias. Este es el quid que permitiría distinguir entre el pluralista del reduccionista, a saber, el pluralista no necesariamente requiere negar los casos de reducción que se han dado históricamente, solamente le basta considerar como no inevitable tal proceso de reducciones y posicionarse conservadoramente respecto a casos de competencia entre T1 y T2 inconmensurables e iguales en su poder explicativo/predictivo sobre cierto $\mathrm{F}$ en cierto instante histórico dado.

Esta actitud conservadora del pluralismo puede ser graduada, en dependencia con lo que se espera suceda con la competencia entre las teorías que versan sobre cierto dominio de fenómenos o sobre cierto $\mathrm{F}$.

Van Bouwel (2014), distingue entre cinco clases de pluralismo epistemológicos: el moderado o temporal, el integrativo, el interactivo, el aislacionista (o de "niveles de análisis"), y el pluralismo "todo vale". Muy concisamente, cada uno se define:

(i) El pluralismo moderado reconoce y promueve la pluralidad temporal de teorías en competencia como medio de alcanzar cierta unidad de las ciencias a largo plazo.

(ii) El integrativo tendría como principio definitorio la integración de múltiples explicaciones las cuales darían cuenta de un mismo fenómeno en sus diferentes niveles.

(iii) La postura del pluralismo interactivo presupone dos puntos; primero, que explicaciones satisfactorias pueden ser obtenidas sin la integración de múltiples niveles, por 
tanto volviendo el imperativo integracionista innecesario y, segundo, que no se debe desalentar la interacción entre niveles, dado que interacción e integración puede llevar a mejores explicaciones.

(iv) La clase aislacionista se opone al integrativo en cuanto que considera cierto privilegio en algún nivel de análisis frente a otros para responder ciertas preguntas, lo cual implica la no necesidad de integración ni de interacción entre niveles, aun cuando considera válidas el conjunto total de las preguntas. Presume cierta "clausura" o autonomía del nivel de análisis.

(v) El pluralismo tipo "todo vale" sería la defensa por retener todas las investigaciones, que emergen de una comunidad científica.

Luego, y en palabras de Van Bouwel:

(...) Puestos en un continuo que va desde el monismo al pluralismo "todo vale", tendríamos monismo, pluralismo moderado, pluralismo integrativo, pluralismo interactivo, pluralismo aislacionista y pluralismo "todo vale". Este ordenamiento refleja una fuerza creciente de la posición pluralista (Jeroen Van Bouwel, 2014: 109).

Considero que el paralelismo que puede establecerse entre el grado de conservadurismo y la división de cinco tipos de pluralismos es patente; si se define que la situación exacta ante la cual el pluralismo aparece como una respuesta posible se produce cuando sobre $\mathrm{F}$ tenemos dos teorías en competencia que comparten similar poder explicativo y predictivo, entonces, en concordancia a las posturas epistemológicas detalladas por Van Bouwel, la situación sería:

(i) Pluralismo moderado: T1 y T2 se deben conservar pragmáticamente hasta que exista el indicio preliminar -por ejemplo, alguna nueva predicción que sea cualitativamente relevante- para decidir entre ambas teorías.

(ii) Pluralismo integracionista: T1 y T2 se deben integrar de modo de lograr con la nueva T3 expandir el poder explicativo/predictivo que tenían T1 y T2 por separado.

(iii) Pluralismo interactivo: T1 y T2 pueden ser integrados o conservados, aun cuando existan algunos indicios preliminares para decidir. 
(iv) Pluralismo aislacionista: T1 y T2 se conservan, dado que F es bien explicado/predicho con la existencia de ambas teorías.

(v) Pluralismo "todo vale": siempre T1 y T2 se conservarán independientemente de cómo vaya evolucionando la investigación sobre F.

\section{Conclusión.}

En esta síntesis conclusiva nos parece oportuno considerar un recuento: el presente artículo en su primera sección, además de exponer algunos antecedentes poco conocidos respecto a cómo las ideas pluralistas ya existían en la primera mitad del siglo XX, así, como también, las nociones de holismo y subdeterminación; presenta sucintamente los argumentos de Suppes (1978), los cuales, a mi parecer, constituyen una perfecta síntesis de las razones para adoptar la postura pluralista. En esta dirección, el pluralismo sería la postura filosófica más compatible con una visión del quehacer de la filosofía de la ciencia alejada de la búsqueda de respuestas totalizadoras y definitivas, es decir, más consciente de sus propios límites. No obstante, nótese que la defensa de esta perspectiva no es el foco del ensayo, sino más bien desarrollar los matices conceptuales del pluralismo, como se planteó en las dos secciones siguientes.

En consecuencia, la segunda y tercera sección constituyen una unidad, ya que parten de la exposición de dos de las grandes problemáticas en filosofía de las ciencias, para desembocar, cada una por su lado, en cierta especificación conceptual respecto a las definiciones de "monismo"/"reduccionismo" y "pluralismo", siendo ambos extremos circunscritos a lo que corresponde a epistemología. La tercera sección rompe el esquema expositivo del resto del artículo, ya que explícitamente ensaya la posibilidad de derivar desde el holismo y la inconmensurabilidad, los rasgos centrales de las clases de pluralismos que se opondrían al (o los) reduccionismo(s). Lo que está desarrollado previamente a esta tercera parte considerada central del artículo, se presenta como una suerte de complemento informativo a bien conocidos sistemas filosóficos de la ciencia, como son los de Kuhn y Feyerabend.

Si bien es cierto que importantes materias han quedado fuera del marco de lo que se ha escrito, como las relaciones entre metafísica y la postura del pluralismo, las relaciones claras que puede establecer con el anarquismo epistemológico -su pluralismo metodológico - ni las ventajas prácticas del pluralismo epistémico frente a los monismos; debo señalar que muchos de tales tópicos requieren un tratamiento un poco más 
específico y de mayor extensión, cuestión que va más allá de los objetivos del presente artículo, cuya meta es ser una exploración conceptual bastante preliminar del tópico. 
Bibliografía

BoKUlich, Alisa (2008). Reexamining the quantum-classical relation. Cambridge University Press.

CARNAP, Rudolf (1931) Überwindung der Metaphysik durch logische Analyse der Sprache. En Ayer, A. J. (1965). El positivismo lógico. México D.F.: Fondo de Cultura Económica.

Dunem, Pierre (1906) Physical Theory and Experiment. En Harding, S. (Ed.). (2012). Can theories be refuted?: Essays on the Duhem-Quine thesis. Springer.

FACUSE, Marisol (2003) Una Epistemología Pluralista. El anarquismo de la ciencia de Paul Feyerabend. Cinta de Moebio. Revista de Epistemología de Ciencias Sociales, 17, 2-16.

Feyerabend, Paul (1975) Against Method: Outline of an Anarchistic Theory of Knowledge. Verso Books.

FODOR, Jerry (1974). Special sciences (or: the disunity of science as a working hypothesis). Synthese, 28(2), 97-115.

Gibson, R. F. (2004) The Cambridge Companion to Quine. Cambridge University Press.

Kellert, Stephen H., Longino, Helen. E., \& Waters, C. Kenneth. (Eds.). (2006). Scientific pluralism. U of Minnesota Press.

Kunn, Thomas S. (1970). The Structure of Scientific Revolutions, 2nd enl5. ed. University of Chicago Press.

Kunn, Thomas S. (1976). Theory-Change as Structure-Change: Comments on the Sneed Formalism. Erkennmis, 10 (1976), 179-199

Neurath, Otto, Carnap, Rudolf, \& Hahn, Hans (1929) Wissenschaftliche Weltauffassung - Der Wiener Kreis. En Revista de Estudios sobre la Ciencia y la Tecnología, Vol. 9, $\mathrm{N}^{\circ} 18$ (2002), 102-149.

NickLEs, Thomas (1973), Two concepts of intertheoretic reduction. The Journal of Philosophy, 70, 181-201.

Osorio, Andrés (2011) La contrainducción o el concepto de relativismo en P. K. Feyerabend. Tesis de Magíster en Filosofía, Universidad de Chile.

Ротоснniк, Angela (2011) A neurathian conception of the unity of science. Erkenntnis, 74(3), 305-319.

Quine, Willard V. O. (1951) Two Dogmas of Empiricism. The Philosophical Review, Vol. 60, No. 1, 20-43.

SuPPES, Patrick (1978) The plurality of science. PSA: Proceedings of the Biennial Meeting of the Philosophy of Science Association, Vol. 1978, 3-16.

VAN BouWEL, Jeroen (2014). Pluralists about pluralism? Different versions of explanatory pluralism in psychiatry. En Galavotti, M. C., Dieks, D., Gonzalez, W. J., Hartmann, S., Uebel, Th. \& Weber, M. (eds.) (2014) New Directions in Philosophy of Science (The Philosophy of Science in a European Perspective Series). Springer 